\title{
Au cœur des nouvelles filières de recyclage des déchets, les ports
}

\author{
Par Juliette CERCEAU \\ Chercheure associée, UMR PACTE \\ Guillaume JUNQUA \\ Maître Assistant HDR, École des mines d'Alès \\ Miguel LOPEZ-FERBER \\ Professeur, École des mines d'Alès \\ et Nicolas MAT \\ Chercheur Doctorant, École des mines d'Alès
}

La transition vers une économie circulaire interpelle les zones portuaires, et ce à plus d'un titre. En tant qu'acteurs majeurs dans l'importation de matières premières, la généralisation de la réutilisation, du recyclage et de la valorisation des déchets peut entraîner une diminution des besoins de ces matières premières. Par contraste, à mesure que cette dynamique se développe, des opportunités de nouvelles activités industrielles apparaissent qui structurent des filières d'approvisionnement et de traitement. Les zones portuaires deviennent des acteurs majeurs dans l'économie des matières premières secondaires, de façon analogue à ce qu'elles sont en ce qui concerne l'économie des ressources naturelles. Ce sont les stratégies à mettre en œuvre pour accompagner cette mutation qui vont conditionner la place que chaque port pourra prendre dans ces marchés.

\section{Les zones industrialo-portuaires (ZIP) exutoires des déchets de leur hinterland}

Les ports et leur domaine maritime ont été pendant longtemps considérés comme des espaces privilégiés pour se débarrasser des déchets. Cela a été vrai tant pour les rejets liquides (via des émissaires les rejetant au large) que pour les déchets solides (transportés par barges ou bateaux puis « clapés » en mer [Ndlr : c'est-à-dire largués sur les fonds marins sans avoir été traités]).

La combinaison de la pression réglementaire, de la valorisation de certains déchets et du besoin des ports en matières premières conduit progressivement à un changement de perspective. Les ports deviennent des plateformes de massification et de transport (hub) de déchets vers d'autres territoires, mais aussi de recyclage de leurs propres déchets et de ceux de leur hinterland, voire même de déchets provenant de l'extérieur.

L'économie des déchets, notamment celle de leur transport (exportation ou importation), dépend du volume à transporter. Ainsi, des filières de traitement des déchets solides ou des rejets liquides produits en grande quantité, surtout s'ils sont difficilement transportables, seront les premières à se structurer. C'est le cas des unités de traitement des eaux usées et de celles traitant des ordures ménagères ou des déchets de construction.

\section{Des ZIP fournisseurs d'utilités}

Les ZIP fournisseurs d'utilités énergétiques

Des unités de valorisation énergétique (UVE) des déchets urbains se sont développées dans de nombreuses ZIP. Ainsi, par exemple, les déchets urbains du territoire du Havre sont collectés et transportés par barges vers une UVE située sur le site industrialo-portuaire de Port Jerôme. Cette unité, Ecostu'air, injecte ainsi près de $100000 \mathrm{MWh} / a n$ dans le réseau électrique (sans compter sa propre consommation). À partir de 2015, une valorisation mixte de l'énergie en vapeur et en électricité sera mise en service.

Dans la ZIP de Fos-sur-Mer, I'UVE exploitée par EveRé reçoit quotidiennement plus de 1000 tonnes de déchets urbains et de boues issues de stations d'épuration. En 2009, elle a produit environ 174000 MWh d'énergie et récupéré 3211 tonnes de métaux à partir des cendres d'incinération.

On trouve également des unités de valorisation des déchets industriels dans d'autres ZIP. Bien qu'il s'agisse surtout d'unités d'incinération des déchets produits par les entreprises situées à proximité (qui représentent en général les apports les plus importants), ces installations peuvent également traiter des déchets provenant d'autres 


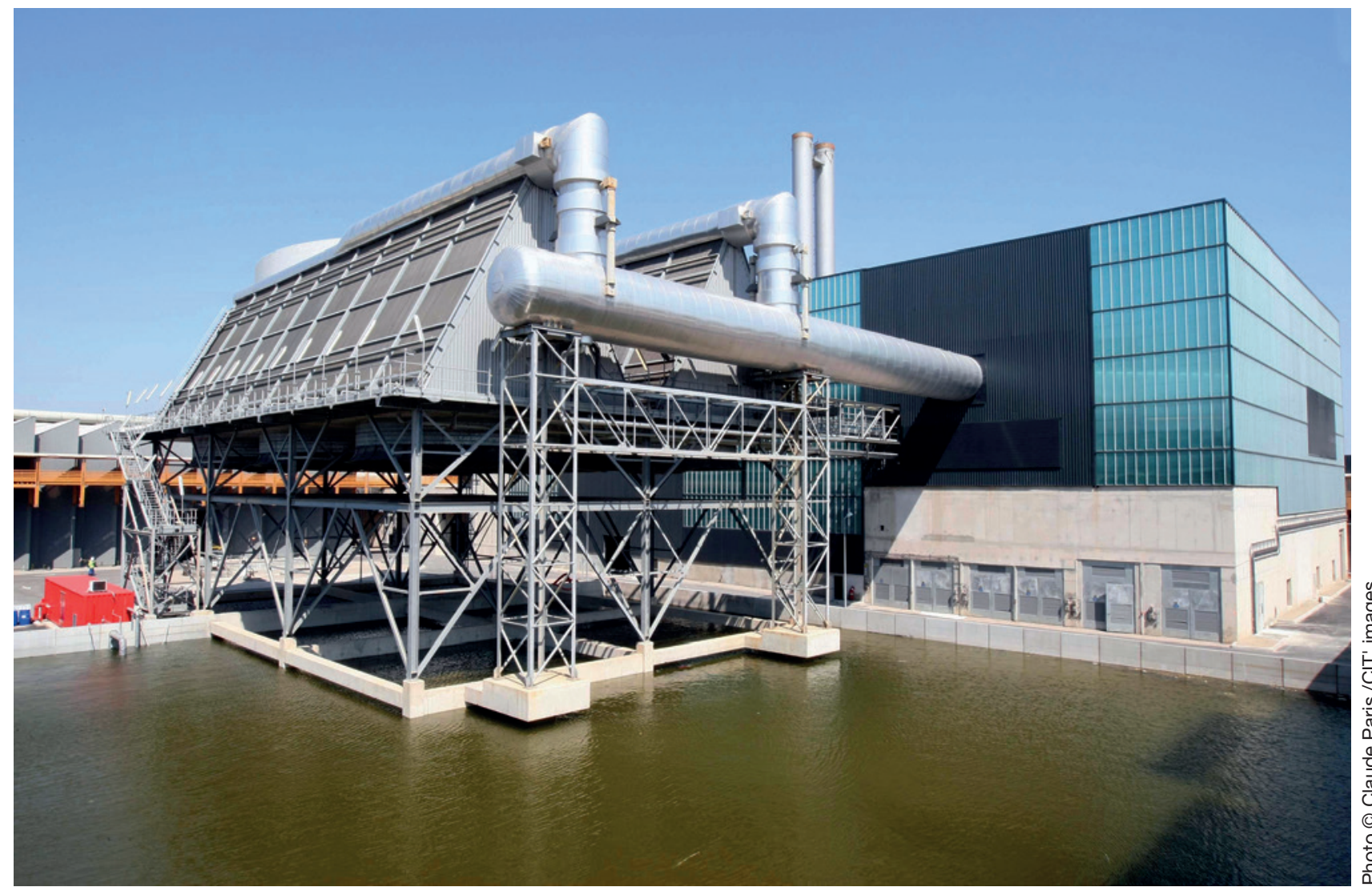

Vue extérieure de l'incinérateur du centre de traitement multi-filières de Fos-sur-Mer géré par le groupe EveRé, mars 2011.

“Dans la ZIP de Fos-sur-Mer, I'UVE exploitée par EveRé reçoit quotidiennement plus de 1 000 tonnes de déchets urbains et de boues issues de stations d'épuration. En 2009, elle a produit environ 174000 MWh d'énergie et récupéré 3211 tonnes de métaux à partir des cendres d'incinération. ”

territoires. L'usine d'incinération et de valorisation de déchets industriels SEDIBEX située dans la ZIP du Havre a une capacité de traitement de 165000 tonnes/an, dont $85 \%$ proviennent des industries normandes. L'énergie (vapeur) produite par Sedibex est ensuite redirigée vers les entreprises de la ZIP via un réseau de chaleur.

À l'étranger, des processus similaires sont à l'œuvre. Par exemple, à Ulsan (Corée du Sud), une politique de transformation des déchets en énergie et matériaux recyclés a été développée dès les années $2000^{(1)}$.

\section{Des ZIP comme fournisseurs d'utilités à l'interface entre l'urbain et l'agricole}

Des initiatives de réutilisation de certains déchets et de certains rejets produits dans les ports fleurissent dans beaucoup de ZIP via la création de synergies non seulement entre des industries, mais aussi avec des zones urbaines (comme, des réseaux de chaleur) ou à destination de l'agriculture. Le cloisonnement entre les activités urbaines industrielles et agricoles - une caractéristique de la fin des années 1990 - tend à s'estomper, et des synergies entre ces différents compartiments apparaissent. Elles permettent la réutilisation de certains effluents difficilement transportables. C'est le cas, par exemple, de l'entreprise Messer (à Lavéra, près de Martigues, dans les Bouches-du-Rhône) qui commercialise du $\mathrm{CO}_{2}$ (120000 tonnes/an) récupéré de procédés pétrochimiques et qui est ensuite purifié pour différents types d'applications (médicales, agroalimentaires ou autres), ou encore le réseau urbain de chaleur de Dunkerque, qui achemine de la chaleur récupérée dans l'aciérie ArcelorMittal.

Un exemple emblématique est le projet $\mathrm{WarmCO}_{2}$, dans la zone portuaire de Zeeland (aux Pays-Bas), qui récupère la chaleur contenue dans les eaux usées et le $\mathrm{CO}_{2}$ émis pour les acheminer vers des serres, augmentant ainsi les rendements de production en biomasse. Un réseau de canalisations d'une longueur de 15 kilomètres a été construit à cet effet. Via ce réseau, $500000 \mathrm{MWh}$ de chaleur et 70000 tonnes de $\mathrm{CO}_{2}$ sont distribués chaque année, permettant l'alimentation de 200 hectares de serres. Des projets d'exploitation du $\mathrm{CO}_{2}$ sont en gestation ou en cours de réalisation dans d'autres zones portuaires, notamment dans celle de Fos-sur-Mer ${ }^{(2)}$.

\section{Les récifs artificiels : une création d'utilités écolo- giques?}

Le développement de récifs artificiels comme moyen de restaurer ou d'augmenter la biodiversité, voire de produire et d'exploiter une biomasse, va en s'accroissant partout

(1) MAT (N.) \& al., 2015, CLEAN (J.), Pro. doi:10.1016/j.jclepro.2015.04.058

(2) http://www.paristechreview.com/2011/12/01/micro-algues-petrole-bleur 
dans le monde. Leur création nécessitent de faire appel à d'importants volumes de matériaux. Ces matériaux peuvent être des déchets de construction, mais aussi d'autres types de structures. Ainsi, des bateaux, des wagons de trains ou encore des structures métalliques ont été immergés après avoir été décontaminés et nettoyés. Cependant, ce sont des blocs de béton qui sont le plus couramment utilisés. Les récifs artificiels peuvent constituer un débouché pour ces filières de recyclage. En France, environ $90000 \mathrm{~m}^{3} \mathrm{de}$ récifs artificiels ont été ainsi immergés, dont un tiers dans la baie du Prado, à Marseille, entre 2007 et 2008. Dans d'autres pays, ces opérations sont d'ampleur beaucoup plus importante (par exemple, le Japon en a installé en 2011 plus de deux millions de mètres-cubes).

\section{Des ZIP pièces maîtresses dans la structuration de filières de traitement des déchets}

\section{L'utilisation des déchets de construction}

Les déchets de construction se caractérisent par leurs importants volumes et par leur faible valeur économique. Leur transport sur de longues distances n'est pas viable économiquement : l'économie de ces déchets est donc plutôt à dimension locale.
Dans le cadre du projet DEPART (3), l'analyse du métabolisme portuaire des flux de déchets a permis d'identifier les principaux gisements de ce type de déchet à l'échelle de l'hinterland portuaire pour deux zones d'étude : le territoire du Havre et celui de Marseille-Fos.

L'activité liée à la transformation de sous-produits industriels (notamment de laitiers de haut-fourneaux) par recyclage est importante, permettant à la fois de réduire les volumes de déchets stockés en centres d'enfouissement technique, mais aussi de diminuer les prélèvements de ressources naturelles, que celles-ci soient locales ou importées.

\section{Les sédiments de dragage}

L'ensablement des ports et des voies de navigation demande des actions périodiques de dragage, qui génèrent des quantités importantes de sédiments (un dragage qui, pour certains ports, peut atteindre sur une année une épaisseur de 0,5 à 1 mètre). La masse des sédiments ainsi extraits représente entre 25 et 45 millions de tonnes de matière sèche par an, pour l'ensemble des ports français. Ces sédiments, qui souvent excédent les limites régle-

(3) MAT (N.) \& al., Rapport final du projet DEPART, ADEME Déchets et Société 2010-2012.

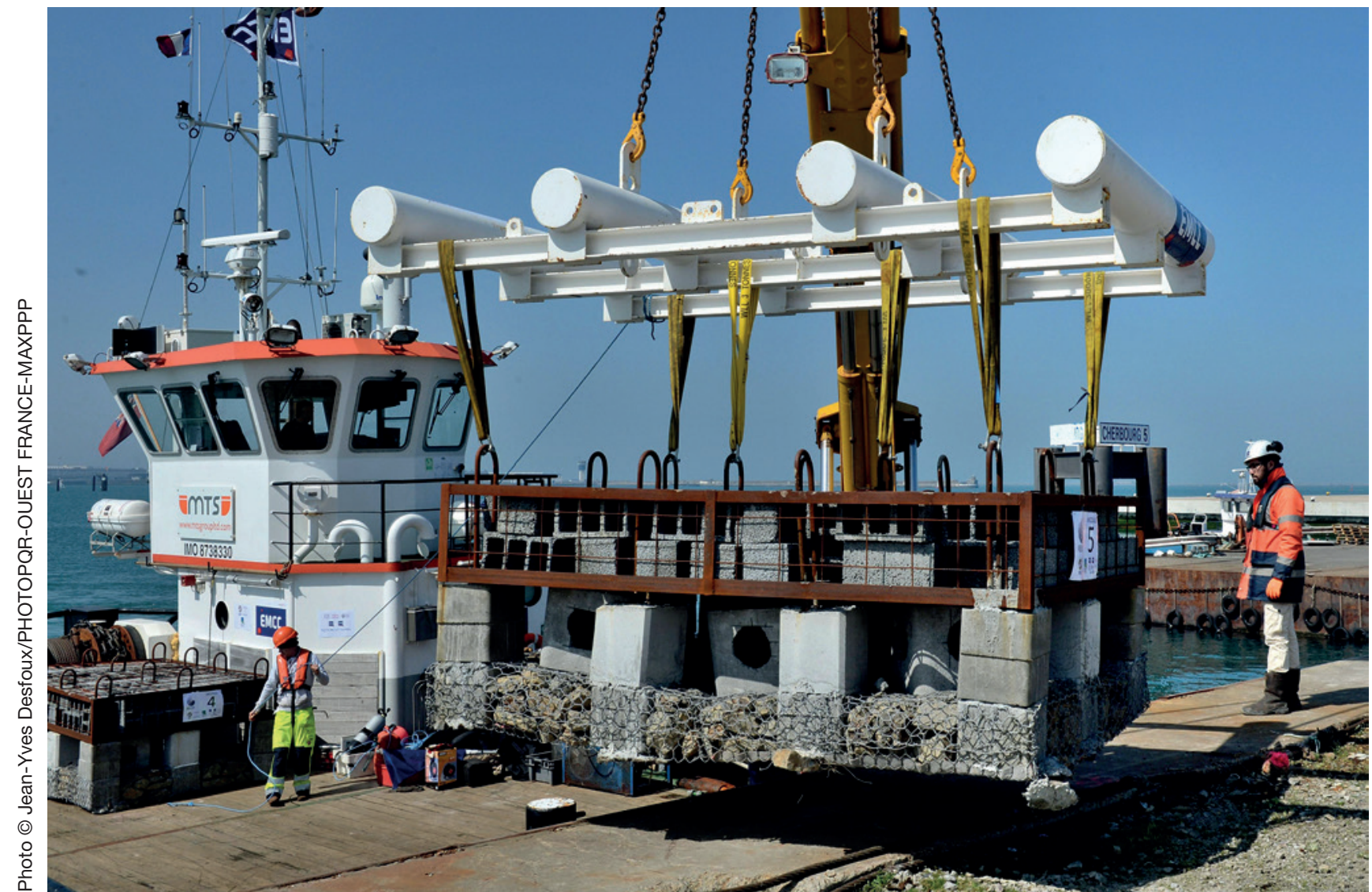

Chargement de récifs artificiels destinés à être immergés dans le cadre du programme européen de coopération transfrontalière France-Angleterre, Cherbourg, avril 2015.

"Le développement de récifs artificiels comme moyen de restaurer ou d'augmenter la biodiversité, voire de produire et d'exploiter une biomasse, va en s'accroissant partout dans le monde. " 
mentaires autorisées en matière de pollution, sont donc considérés comme des déchets, une fois à terre. Cependant, la pollution se concentre seulement dans certaines fractions granulométriques, permettant, si un tri approprié est fait, une réutilisation des fractions non polluées.

Des filières de valorisation des sédiments de dragage sont en cours de développement au niveau des grands ports commerciaux et de ports de plaisance. Le projet Ecodredge-med (4) a eu comme objectif la valorisation de ces sédiments notamment pour servir au ré-ensablement des plages et à la protection de la ligne de côte. Par ailleurs, dans le nord de la France, la chaire Ecosed (5) vise également à trouver des voies de valorisation des sédiments dragués dans le port de Dunkerque pour des applications dans le secteur de la construction et des travaux publics, notamment en substitution directe à des granulats provenant de sources conventionnelles.

\section{Les déchets de navires}

Les zones portuaires proposent, notamment en Europe, des services de vidange des fonds de cale de navires, des eaux de ballast et des eaux sanitaires, ainsi que la collecte des déchets solides (plastiques alimentaires et autres). Certains de ces flux (notamment les eaux de fonds de cale) peuvent être plus ou moins chargés en hydrocarbures. Toutefois, même si des acteurs se positionnent progressivement sur ce secteur du service aux navires dans le traitement de leurs déchets et si des solutions techniques sont mises en œuvre à cette fin dans les différents ports, il convient de noter que la collecte et le traitement de ces déchets ne sont pas harmonisés d'une place portuaire à l'autre ni au niveau des redevances payées par le propriétaire du navire ni au niveau de la valorisation possible de ces déchets. Le renforcement et la mise en application des conventions internationales visant à empêcher les dégazages en mer devraient augmenter ces flux de déchets à gérer à terre.

En 2010, les déchets d'exploitation des navires ont représenté $172000 \mathrm{~m}^{3}$ pour le seul Grand port maritime de Marseille, dont plus de $80 \%$ ont été recyclés. La structuration de filières locales de valorisation (récupération, séparation et revalorisation énergétique) et la coordination entre les acteurs sont des éléments capitaux pour permettre une création de valeur économique. Ainsi, la société RTDH s'est spécialisée dans la récupération et le traitement d'eaux de navires chargées en hydrocarbures. Ces hydrocarbures, une fois séparés, peuvent ensuite être valorisés énergétiquement en interne, ou commercialisés pour des usages en tant que valorisation matière ou pour des usages énergétiques.

\section{Le démantèlement de navires}

Entre 200 et 600 navires de plus de 2000 tonnes de port en lourd (TPL) sont démantelés chaque année en France, dont environ $18 \%$ battant pavillon d'un autre pays européen. Durant les dernières années, le démantèlement de ces navires a diminué en Europe, conduisant à un flux de matières vers des pays tiers, comme l'Inde ou le Bengladesh, qui récupèrent près de $90 \%$ de l'activité mondiale. Étant donné le potentiel économique et les enjeux envi- ronnementaux qui sont associés au démantèlement des navires, l'État français souhaite développer des filières de démantèlement et de recyclage permettant un traitement dans de bonnes conditions des matériaux dangereux pouvant se trouver à bord des navires à démanteler, mais aussi le recyclage d'autres composants (notamment les métaux composant les moteurs ou les coques).

La déconstruction de navires (plus petits) de plaisance ou de pêche présente elle aussi d'importants enjeux. La construction des coques en matériaux composites (fibre de verre ou résines) implique certains enjeux techniques pour leur recyclage du fait notamment de la difficulté de procéder à la séparation des fibres composites. La structuration de filières ad hoc en France est à l'étude.

\section{Les ZIP convoyeuses de matières premières et secondaires}

\section{Le marché des matières premières secondaires}

Dans les exemples vus précédemment, les déchets traités avaient des origines souvent proches et la distance couverte par leur transport restait limitée. On observe toutefois une augmentation de l'utilisation de ressources secondaires (matières premières secondaires) dans le secteur industriel. Ces ressources ne proviennent plus du seul traitement des déchets de l'hinterland du port concerné : ils s'inscrivent dans un marché global, celui du transport de matières. Dans les zones de traitement, la transformation de ces déchets en ressources fait appel au développement de filières de recyclage spécialisées qui peuvent structurer autour d'elles tout un écosystème industriel.

En 2012, la France a importé 3,47 millions de tonnes de déchets et en a exporté un peu moins de 9 millions de tonnes. II s'agissait principalement de métaux (80\% des importations et $71 \%$ des exportations). Le flux économique généré par l'exportation de matières premières de recyclage est évalué à 4,4 milliards d'euros pour 2012, et à 2,7 milliards d'importations correspondant à 15 millions de tonnes de matières recyclées. Ainsi, $41 \%$ de l'acier utilisé provient du recyclage, et le taux est un peu plus élevé en ce qui concerne les métaux non ferreux (46\%). Ce recyclage se traduit par une économie d'un peu plus de 12 millions de tonnes de ressources primaires de fer et de 2,5 millions de tonnes de métaux non ferreux non consommées ${ }^{(6)}$.

Sans revenir sur la gestion de la fin de vie des navires évoquée supra (voir le paragraphe «Démantèlement des navires »), nous présenterons l'exemple de la filière DEEE (déchets d'équipements électriques et électroniques).

\footnotetext{
(4) Projet Ecodredge-Med (FUI, 2011).

(5) http://www2.mines-douai.fr/actualites/mines-douai-cree-unechaire-industrielle-de- recherche-sur-les-sediments.

(6) ADEME 2014, Déchets - Chiffres-clés.
} 


\section{Le recyclage des DEEE}

La miniaturisation des équipements électroniques et les avancées technologiques rapides en la matière exigent la mise en place de filières de haute technologie capables de s'adapter rapidement aux changements dans les composants électriques et électroniques. Malgré ces contraintes, le gisement disponible est très important : en Europe, la moyenne de DEEE par habitant a été d'un peu plus de $19 \mathrm{~kg}$ en $2012^{(7)}$, soit un gisement de 1,3 million de tonnes, et ce seulement pour la France. Ces appareils contiennent d'importantes quantités de métaux rares et précieux, ainsi que de matières plastiques.

Avant la structuration de filières spécialisées, ces équipements étaient majoritairement exportés via quelques ports spécialisés (notamment Chennai et Mumbai, en Inde, et Singapour ou Nanhai, en Chine) et arrivaient en Chine, en Inde ou au Pakistan pour y être traités. Ces trois pays représentaient plus de $90 \%$ du marché mondial en 2002.

La directive européenne 2002/96/CE, transposée en France par le décret n`2005- 829 du 20 juillet 2005 (avec des actualisations intervenues en 2012 et 2014), a fixé le cadre du traitement de ces déchets, impliquant une collecte séparée spécifique permettant de favoriser leur réemploi, leur réparation en vue d'une réutilisation, et le recyclage et la valorisation de ces déchets, mais aussi, en agissant dès la conception de ces équipements, de réduire les quantités de déchets toxiques. La mise en place des filières de responsabilité élargie du producteur (REP) a permis une meilleure traçabilité des produits et des matériaux les constituant. Aujourd'hui, la structuration des filières ad hoc en France a permis le développement d'activités de recyclage de DEEE sur le territoire national et un arrêt de leur exportation. En France, 453689 tonnes de DEEE ménagers et 23200 tonnes de DEEE professionnels ont été déclarées et traitées en 2013. Plus de $99 \%$ sont traités en France, et plus de $78 \%$ de leurs matériaux ont été recyclés.

\section{Conclusion}

À mesure que l'économie des déchets et leur valorisation se développent, de nouvelles opportunités s'ouvrent aux territoires portuaires non seulement en tant que hubs de transport, mais aussi en tant que centres de traitement, de transformation et de récupération de matières à haute valeur rajoutée.

En raison de la complexité des chaînes de retraitement, un positionnement en tant qu'acteur présuppose la structuration d'une filière complète d'industriels dans le cadre d'un projet de territoire. C'est l'ambition de la région Nord-Pas-de-Calais, qui vise à devenir une place de leader en Europe en matière de récupération de métaux stratégiques et de terres rares nécessaires à la fabrication d'équipements électriques et électroniques. Cela implique la structuration d'une filière de collecte qui devra sans doute dépasser les frontières nationales et très certainement impliquer les zones portuaires. De forme similaire, d'autres zones portuaires pourront se spécialiser dans le traitement d'autres déchets ou la récupération d'autres matières premières secondaires.

(7) ADEME 2013, Équipements électriques et électroniques, Collection "Repères". 